\title{
Time-critical Underwater Sensor Diffusion with No Proactive Exchanges and Negligible Reactive Floods
}

\author{
Uichin Lee*, Jiejun Kong, Mario Gerla \\ Department of Computer Science, University of California, Los Angeles \\ Joon-Sang Park \\ Department of Computer Engineering, Hong-Ik University \\ Eugenio Magistretti \\ Dipartimento di Elettronica, Informatica e Sistemistica, Università di Bologna
}

\begin{abstract}
In this paper we study multi-hop ad hoc routing in a scalable Underwater Sensor Network (UWSN), which is a novel network paradigm for ad hoc investigation of the world below the water surface. Unlike existing Underwater Acoustic Networks (UAN), the new UWSN paradigm dispatches large number (in the thousands) of unmanned low-cost sensor nodes to locally monitor and report otherwise not easily accessible underwater events in a time-critical manner. Due to the large propagation latency and very low bandwidth of the acoustic channel, a new protocol stack and corresponding models are required as conventional approaches fail. In particular, we show that neither proactive routing message exchange nor reactive/on-demand flooding is adequate in the challenging new underwater environment. Unlike the terrestrial scenarios, on-demand flooding cannot be both reliable and efficient due to widespread collisions caused by the large propagation delay. On the other hand, as in terrestrial scenarios, proactive routing is more expensive and less efficient than on-demand routing in typical underwater environments. We propose a "conservative" communications architecture that minimizes the number of all packet transmissions to avoid possible acoustic collisions. This is implemented in the non-intrusive Under-Water Diffusion (UWD), which is a multi-hop ad hoc routing and in-network processing protocol with no proactive routing message exchange and negligible amount of on-demand floods. To achieve its design goal, UWD does not rely on GPS or power hungry motors to control currents. Instead, UWD is designed in a minimalist's framework, which assumes homogeneous GPS-free nodes and random node mobility. Our simulation study verifies the effectiveness and efficiency of our design.
\end{abstract}

Key words: Underwater Sensor Network, Routing Protocol 


\section{Introduction}

The still largely unexplored vastness of the ocean, covering about two-third of the surface of earth, has fascinated humans for as long as we have records for. For the past several centuries, the ocean has played an increasingly important role in transportation and military activities. In emergent event investigations, for example, for marine incidents (cargo ship sinking involved with chemical pollution and oil spill), military demands (submarine tracking) and underwater research (exploration of underwater volcano eruption), the state-of-the-art in communication technology has significantly surpassed the state-of-the-art of physical investigation in regard to effectiveness and efficiency. This calls for the need of building a large-scale short-term and distributed data acquisition network for time-critical aquatic applications.

We envision that a large-scale underwater sensor network (UWSN) is the answer to support these time-critical aquatic applications. A large amount of underwater sensor nodes can be airdropped to the venue immediately after the incident. An area of hundreds of square nautical miles may need thousands of sensor units. At real time, each ad hoc sensor unit monitors local underwater activities and reports sensed data via multi-hop acoustic routes to a distant command center (i.e., the network sink). Clearly, the advantages of the new UWSN paradigm are: (1) Localized and coordinated sensing is far more precise than the current remote telemetry technology, e.g., those relying on long-range directional frequency and ranging (DIFAR) sonobuoys. (2) Scalability of UWSN ensures that a large area can be covered for time-critical applications. (3) Underwater hazardous environment is expected to have negligible impact on human operators when unmanned electronic platforms are used below the water surface. (4) The time-critical sensor deployment is low-cost. A low-cost underwater sensor unit is un-powered and flows by the water current to sense and harvest data in an ad hoc manner.

The new UWSN paradigm, however, poses formidable new challenges compared to the existing wireless radio sensor networks. In contrast to wired networks, wireless radio networks operate in a resource constrained environment. Based upon technology for dense wave division multiplexing (DWDM), a single optical bundle can carry $12,800 \mathrm{GHz}$ of optical signal. However, even the richest frequency band owner in the United States, namely DoD (Department of Defense), only owns approximately $300 \mathrm{MHz}$ of the total $3 \mathrm{GHz}$ of useable radio spectrum. Of the $300 \mathrm{MHz}$ owned by the military, individual systems are allocated in small blocks, e.g. $10 \mathrm{MHz}$, $1 \mathrm{MHz}$, or less. Consequently, protocols for wireless radio sensor networking must be far more efficient than protocols for wired networks. The wireless radio networks demand several orders of magnitude improvement in protocol efficiency compared to their wired counterparts.

Nevertheless, if we extend our vision to the underwater world, then wireless radio networks become the one with relatively much richer resource to expend. As high-frequency signals are quickly absorbed in water, underwater networking must rely on low-frequency acoustic communication, with the frequency upper bound reported as $1 \mathrm{MHz}$ at 60 -meter range [7]. This implies that the entire acoustic band is less than several $\mathrm{MHz}$ and typical allocation is measured in $\mathrm{KHz}$ for individual systems. This drastic reduction in communication resource makes underwater networking an extremely challenging topic. The underwater wireless acoustic networks demand

\footnotetext{
* Corresponding author. Phone: +1-310-825-4367, Fax: +1-310-825-2273, Address: Boelter Hall 3803, Department of Computer Science, UCLA, Los Angeles, CA90095

Email addresses: $\{$ uclee, jkong, gerla\}@cs.ucla.edu (Uichin Lee*, Jiejun Kong, Mario Gerla), jspark@cs.ucla.edu (Joon-Sang Park), emagistretti@deis.unibo.it (Eugenio Magistretti).
} 
several orders of magnitude improvement in protocol efficiency compared to their wireless radio counterparts.

Therefore, in order to realize the demanding UWSN paradigm, new models and protocols are required in most layers. One area which will definitely require revisiting (with respect to prior work in wireless radio networks) is multi-hop packet delivery in a wireless acoustic network with random node mobility and without GPS support. This will be the main focus of our paper. Below, we give a revisit of on-demand flooding and proactive routing message exchange in GPS-free multi-hop ad hoc networks.

First, position is important in underwater sensor nets. So far, to our best knowledge, a scalable and low-cost positioning system like GPS is not yet available underwater. One must rely on multi-hop GPS-free localization schemes [2] to let the sensor nodes know their positions. Second, either flooding or network-wide packet exchange is needed in multi-hop ad hoc networking. Excluding geo-routing, multihop routing protocols fall into two categories: proactive routing and reactive routing (aka., on demand routing) [1]. In proactive ad hoc routing protocols like OLSR, TBRPF and DSDV, mobile nodes in all network areas constantly exchange routing messages which typically include connection status to other nodes (e.g., link state or distance vector), so that every node maintains sufficient and fresh network topological information to allow them to find any intended recipients at any time. On the other hand, on demand routing has become a major trend in dynamic ad hoc networks. AODV [15] and DSR [5] are common examples. Unlike their proactive counterparts, on demand routing operation is triggered by the communication demand at sources. Typically, an on demand routing protocol has two components: route discovery and route maintenance. In route discovery phase, the source seeks to establish a route towards the destination by flooding a route request (RREQ) message, then waits for the route reply (RREP) which establishes the on-demand route. In the route maintenance phase, nodes on the route monitor the status of the forwarding path, and report to the source about route errors. Optimizations could lead to local repairs of broken links.

Finally, flooding and network-wise packet transmission are required in data-centric protocols like Directed Diffusion [4]. The network command center (i.e., sink) periodically issues "interests" that are disseminated towards qualified sensor nodes in designated areas. This requires network-wide or at best scope constrained flooding. In addition, if sensor nodes are mobile (e.g., atmospheric sensors carried by weather balloons), both on-demand flooding and proactive routing incur extra overhead to address dynamic network topology changes.

In this paper, we study the impact of the acoustic environment on conventional ad hoc and sensor protocols described above. We show that such protocols are no longer effective and efficient under water. The following observations are critical for realistic-underwater sensor network design:

(i) Ineffectiveness and inefficiency of flooding. Deployment redundancy is an innate characteristic of ad hoc networking to avoid network partitioning. Flooding a message in such a redundant ad hoc network is normally considered as a reliable operation that reaches every ad hoc node. Unfortunately, by analytic and simulation study we show that this is no longer true in underwater sensor networks. In contrast, each network flood cannot be both effective (i.e., delivered to nearly all ad hoc nodes) and efficient (i.e., with low latency and transmission cost). Since flooding is needed in GPS-free on-demand ad hoc routing, this dilemma poses great challenge to multi-hop packet delivery service in a dynamic underwater sensor network.

(ii) Ineffectiveness and inefficiency of proactive routing message exchange. In many situa- 
tions [1][13][15] on-demand routing protocols have been demonstrated to perform better with significantly lower overheads than proactive routing protocols. In this paper we also show that underwater proactive routing fails due to heavy packet collision loss. Unlike radio networks, since the collision loss is mainly caused by signal propagation delay in acoustic networks, reducing packet size does not necessarily reduce the chance of collision to gain better protocol performance. Therefore, proactively exchanging long or short routing messages under the water inevitably generates acoustic traffic that disrupts any routing protocol's performance.

To answer the new challenge, we propose to minimize the number of all packet transmissions to avoid possible acoustic collisions. This includes prohibiting proactive routing message exchange and minimize the total number of on-demand flooding trials. These requirements are implemented in Under-Water Diffusion (UWD) described in Section 3, which is a multi-hop ad hoc routing and in-network processing protocol with no proactive routing message exchange and negligible amount of on-demand flooding trials. Our analytic study defines and proves the "negligibility" constraint, and our simulation study verifies the effectiveness and efficiency of our design.

The paper is organized as follows. Section 2 presents the problem statement. It explains why on-demand flooding and proactive routing fail or have to be inefficient in underwater networks. In Section 3 we present the design details of UWD followed by the analysis in Section 4 . The simulation study shown in Section 5 verifies the effectiveness and efficiency of UWD. Section 6 describes related work. Finally Section 7 concludes the paper.

\section{Problem Statement}

In this section, we first describe the design assumptions and the typical data-centric packet delivery service in GPS-free terrestrial sensor network, using directed diffusion as the example. Then we show the reason why the existing approaches fail in GPS-free underwater sensor networks.

\subsection{Design assumptions}

Underwater acoustic (UW-A) channel Communications in the UnderWater Acoustic (UW-A) channel are with two innate characteristics: narrow \& low bandwidth and very large propagation latency.

The available bandwidth of the UW-A channel is limited and strongly depends on both range and frequency. UW-A channel's acoustic band is limited due to absorption and most acoustic systems operate below $30 \mathrm{kHz}$ [7]. This fact has two significant impacts on underwater communication. First, the entire width of underwater acoustic frequency band is very narrow, so far the highest value reported is around $1 \mathrm{MHz}$ spectrum at the range of $60 \mathrm{~m}$ radius [6]. The entire width of useful acoustic bands is only a small fraction of useful RF bandwidth. Therefore, compared to radio networks, where narrow-band interference can be ameliorated by spread spectrum technology etc., underwater communications do not have an analogous answer. Second, as surveyed in [7], research system or commercial system have highly variable link capacity and the attainable range $\times$ rate product can hardly exceed $40 \mathrm{~km}-\mathrm{kbps}$. Long-range acoustic signal that operates over several tens of kilometers may have a capacity of only several tens of bits per 
second, while a short-range system operating over several tens of meters may have several tens of kilobits per second. Compared to radio or wired links, in both cases bit rates are significantly lower.

The signal propagation speed in the UW-A channel is only $1.5 \times 10^{3} \mathrm{~m} / \mathrm{sec}$, which is five orders of magnitude lower than radio propagation speed $3 \times 10^{8} \mathrm{~m} / \mathrm{sec}$ in the air. The incurred huge latency exceeds the counterpart values in satellite radio communications. For example, the signal propagation latency between an underwater transmitter and a receiver that are 2 kilometers apart is comparable to the one between the earth and the moon in radio transmission. This huge propagation delay has great impact on network protocol design. As the huge end-to-end round trip time (RTT) becomes the performance bottleneck, many common network protocols do not work as expected if they are directly ported from radio networks.

Network assumption Each UWSN node should be a low-cost embedded system equipped with necessary sensing devices. Due to water current and other underwater activities, underwater sensor nodes, except those nodes closely mounted on the sea floor, are with random mobility at a low or medium speed. From empirical observations, underwater objects may move at the speed of 2-3 knots (or $1.0-1.5 \mathrm{~m} / \mathrm{s}$ ) in a typical ocean current condition.

An UWSN has at least one command center (sink) which disseminates commands to the network and meanwhile collects sensing data from the network. Except this imperative centralized control, the other components of the UWSN are tetherless and self-organizing.

We assume that network is dense enough such that there is no partition in the network and there is sufficient redundancy of paths between the sources and sink. This implies that in a network locality there are usually some redundant network members.

At physical layer, currently we assume omni-directional acoustic transmission and reception. Directional transmission and reception will be addressed in future work. We assume that majority of underwater nodes are connected with tetherless acoustic links, rather than wired links. In terms of both deployment and maintenance, it is relatively hard to deploy and maintain multiple underwater nodes intertwined by wires.

\subsection{Flooding Dilemma below the water surface}

To show the problem of flooding, we must at first make some assumptions on the MAC layer design. First, it is expected that multiple acoustic transmitters will employ FDMA (using orthogonal frequencies), CDMA (using orthogonal Walsh codes) and/or MIMO (using orthogonal space-time coding) to share the same medium with "no collisions" during the same time period. We assume that underwater sensor nodes will have these capabilities in the foreseeable future. Yet, an increasing number of multiple transmissions during the same time period will eventually exhaust the channel's degrees of freedom. When FDMA/CDMA/MIMO finally reaches its optimality bound, a contention avoidance method must be used at the MAC layer to meet the demand.

On top of FDMA/CDMA/MIMO, it is possible to implement time division multiple access (TDMA) or random access protocols (e.g, CSMA, ALOHA and slotted ALOHA) as the contention avoidance method. However, carrier sensing is not effective in the UW-A channel due to long propagation delay, and thus CSMA may not be viable. The lack of CSMA increases the vulnerable interval in under water acoustic MAC by orders of magnitude with respect to radio based MAC protocols. The usual remedy to overcome this problem is TDMA type transmission (like in 
satellite channels). TDMA requires time synchronization, but in underwater fine grain synchronization (which is required for time critical applications) is impossible due to large propagation delay and mobility of nodes [20]. Thus, transmissions must be randomly scheduled over very large intervals to achieve reasonable chance of success over a multi hop path, which in turn leads to very low throughput. Therefore, in this paper we choose "pure ALOHA" as the contention avoidance method in our analytic and simulation study.

Suppose an ALOHA node $B$ starts transmission before the last bit from a node $A$ arrives at $B$, collision occurs and both transmissions are dropped in the colliding area. The time of collision occurrence $\left(T_{c}\right)$ must be

$$
T_{c}=T_{x}+T_{p}
$$

where $T_{x}$ and $T_{p}$ are transmission time and one-hop propagation latency, respectively. ${ }^{1}$ Two transmissions will not collide if their starting moments are separated for more than $T_{c}$ long. In radio networks, $T_{p}$ can be ignored within short distance, so $T_{c}=T_{x}$. But in underwater networks, we will show that the term $T_{p}$ is now a decisive factor.

For the ease of analysis, let's study ALOHA in the unit-disk transmission model. Acoustic transmissions within the one-hop disk of radius $R$ are received by the recipients, or the packet is dropped. Let $v=1500 \mathrm{~m} / \mathrm{s}$ denote the propagation speed of underwater acoustic signals.

$$
T_{c}=T_{x}+\frac{R}{v}
$$

As surveyed in [7], the underwater $T_{x}$ depends on the communication range and usually is presented as the bound of range-rate product (i.e., $40 \mathrm{~km}-\mathrm{kbps}$ according to [7]). Let $\ell_{b}$ denote the size of a packet, and then $T_{x} \approx \frac{R \cdot \ell_{b}}{40 k m-k b p s}$. Then we have

$$
T_{c} \approx R \cdot\left(\frac{\ell_{b}}{40 k m-k b p s}+\frac{1}{1500 m / s}\right)
$$

In IP networks, a TCP/UDP packet is less than $\approx 1500$ bytes long, then the transmission time is at most $\frac{\ell_{b}=1500 \cdot 8-b i t}{40 \mathrm{~km} \text {-kbit } / \mathrm{s}}=3 \cdot 10^{-4} \mathrm{~s} / \mathrm{m}$. This maximal value is significantly smaller than the counterpart propagation latency $1 /(1500 \mathrm{~m} / \mathrm{s})=6.67 \cdot 10^{-4} \mathrm{~s} / \mathrm{m}$. In a nutshell, $T_{p}$ dominates $T_{x}$ below the water surface.

Let's assume that, in order to avoid collision, each node must wait at least $T_{b}$ time to transmit next packet. Clearly, $T_{b}$ must be greater than $T_{c}$ to make the collision avoidance strategy work. Let $m$ denote the value $\frac{T_{b}}{T_{c}}$, which is similar to the back-off window size in some collision avoidance MAC protocols. We want to see how the value of $m$ affects the probability of a successful packet transmission with no collision.

This problem can be mapped into the classic occupancy problem [14]. Given that there are $n$ balls (i.e., contending transmissions) and $m$ bins (i.e., collision avoidance window size), we can derive $P_{c}$, the probability of at least one coincidence (i.e., two balls collide into the same bin):

$$
P_{c}(m, n)=1-\frac{m^{(n)}}{m^{n}}
$$

where for positive integers $m, n$ with $m \geq n$, the number $m^{(n)}$ is defined as $m^{(n)}=m(m-$ 1) $(m-2) \cdots(m-n+1)$.

When $n$ is less than $\sqrt{m}$, and $m$ is sufficiently large,






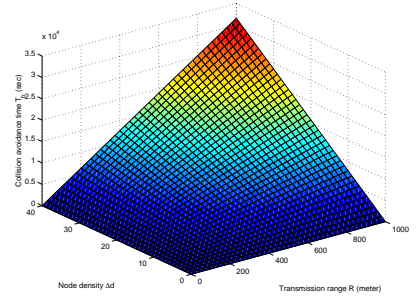

Fig. 1. Distribution of contention avoidance time with $\alpha=0.5$

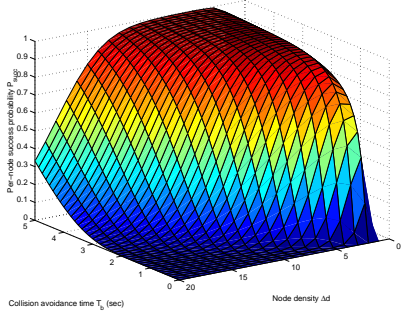

Fig. 2. Per-node success probability $P_{\text {succ }}$ for a flood (transmission range $R=100 \mathrm{~m}$ )

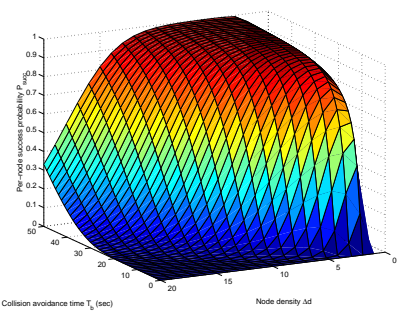

Fig. 3. Per-node success probability $P_{\text {succ }}$ for a flood (transmission range $R=1000 \mathrm{~m}$ )

$$
P_{c}(m, n) \simeq 1-e^{-\frac{n^{2}}{2 m}}
$$

Thus the probability of a transmission with no collision:

$$
P_{0}=1-P_{c} \simeq e^{-\frac{n^{2}}{2 m}} .
$$

In practice, $P_{0}$ must be larger than certain value $P_{0} \geq \alpha$, e.g., $\alpha=0.5$ to ensure that packets are more likely to be received without collision rather than dropped in the contended channel. The referential $\alpha$ is the value used in radio networks (where $T_{x}$ dominates $T_{p}$ ), and

$$
m \geq \frac{n^{2}}{-2 \ln \alpha} .
$$

Let $\Delta d$ denote the average node density in the network (i.e., an average node has $\Delta d-1$ neighbors within its one-hop communication range). $\Delta d=n$ is the number of contending transmissions in our analysis. The required collision avoidance time

$$
T_{b}=m \cdot T_{c} \geq \frac{\Delta d^{2}}{-2 \ln \alpha} \cdot\left((3+6.67) R \cdot 10^{-4}\right)
$$

Figure 1 shows the distribution of the number of slots according to Equation (2). Clearly, for any constant $\alpha$, the appropriate collision avoidance time increases rapidly as the communication range $R$ or average density $\Delta d$ grows.

Now let's study how network floods are affected. Network floods in ad hoc networks are implemented by un-ACKed wireless broadcasts. Such a broadcast transmission is lost due to collision with no link layer automatic re-transmission (ARQ) support. Unfortunately, the chance of collision is non-negligible unless we use a huge collision avoidance window size $m$. Let's analyze how the settings of parameter $m$ and $\Delta d$ affect the quality of flooding.

During a network flood, the maximal number of flooded packet received on an average node is the number of its neighbors $\Delta d-1$. The flooded packet fails to reach the average node if all these packets are lost due to collision. Thus with per-node failure probability $P_{\text {fail }}$, the flooded packet fails to reach an average node. $P_{\text {fail }}$ is computed on the failure probability of all $\Delta d-1$ transmissions:

$$
P_{\text {fail }}=\left(1-P_{0}\right)^{\Delta d-1}=\left(1-e^{-\frac{\Delta d^{2} \cdot T_{c}}{2 T_{b}}}\right)^{\Delta d-1}=\left(1-e^{-\frac{\Delta d^{2} \cdot\left(9.67 R \cdot 10^{-4}\right)}{2 T_{b}}}\right)^{\Delta d-1}
$$




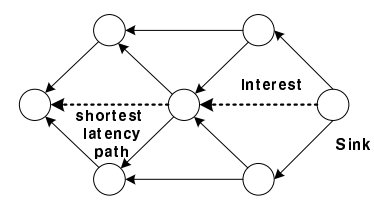

(a) Initial Interest Dissemination

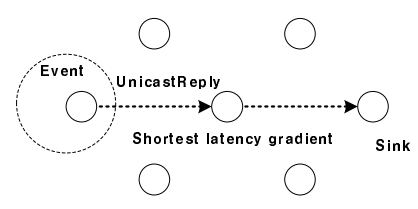

(b) Detecting an event within bounded time and then setting up a path

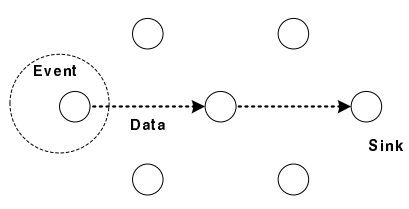

(c) Event report

Fig. 4. Under-Water Diffusion: Immediate Report Protocol (IRP)

As a result, with per-node success probability $P_{\text {succ }}=1-P_{\text {fail }}$, the flooded packet can reach an average node.

Figure 2 shows how $P_{\text {succ }}$ is affected by the collision avoidance time $T_{b}$ and one-hop transmission range $R$. Figure 3 shows that the collision avoidance time $T_{b}$ increases a scale (10 times) if the transmission range $R$ increases a scale. In particular, given a reasonable node density range $[5 . .20]$ and reasonable transmission range $[200 . .1000] \mathrm{m}$, the collision avoidance time $T_{b}$ must be several seconds to guarantee a successful flood. Both figures show that a flooding process is inefficient in latency, thus the entire flooding process is very slow. If the sensor nodes are moving in a scalable network (which implies more hop counts), then the overall flooding delay increases proportionally to the slow $T_{b}$ and the network diameter. This implies more stale routing status and more route outages. On the other hand, if we want to limit the collision avoidance time $T_{b}$ to implement a faster flood, then the success probability $P_{\text {succ }}$ is exponentially reduced towards zero when the node density or transmission radius increases linearly.

In our analysis, the failure probability is computed in every one-hop neighborhood. Thus it doesn't matter whether the flood is a network-wise flood or a limited-range controlled flood (e.g., using a Time-To-Live field to limit the hop count). A flood fails with non-negligible probability in the intended neighborhoods. In a nutshell, a flood in UWSN cannot be both efficient and reliable. Therefore, if flood is ever used in an UWSN protocol, the network incurs major overhead to make each flood work and thus achieves very poor efficiency.

\section{Design}

In this section we propose Under-Water Diffusion (UWD), a multi-hop ad hoc routing and innetwork processing protocol with no proactive routing message exchange and negligible number of on-demand floods. It is designed in a minimalist's framework, which assumes homogeneous GPS-free nodes and random node mobility.

\subsection{Design Principles}

The UWD protocol design is non-intrusive. It is guided by the following design principles.

No proactive routing message exchange. In the UW-A channel, the cost of proactive packet exchange is more expensive than its counterpart in radio networks. In radio networks, channel contention can be ameliorated by using small-size packets (to reduce packet transmission delay $T_{x}$ ). Unfortunately, reducing transmission delay $T_{x}$ is less useful below the water surface because 


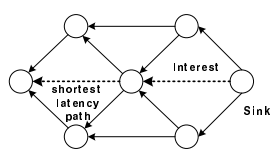

(a) Initial Interest Dissemination

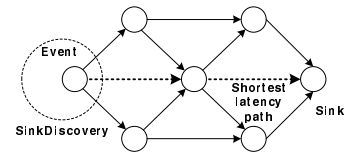

(b) Upon detecting an event, a SINKDISCOVERY message is flooded to find route towards the sink

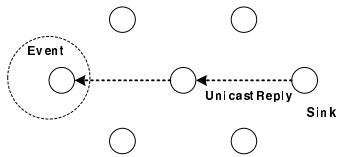

(c) A sink replies back an UNICASTREPLY message and using this each node sets up a path

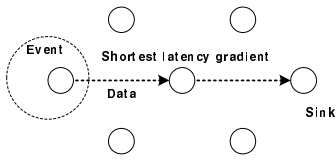

(d) Event report

Fig. 5. Under-Water Diffusion: Delayed Report Protocol (DRP)

propagation latency $T_{p}$ is now a dominant factor that affects the channel condition. The total number of transmissions is a more important metric now.

Proactive routing incurs network-wise transmissions per proactive interval $T_{d}$. Clearly, proactive routing incurs various amount of transmissions if $T_{d}$ varies. However, in a mobile underwater network like UWSN, a larger $T_{d}$ means more stale routing state, while a smaller $T_{d}$ means more severe channel contention. In contrast, in our UWD design, multi-hop acoustic paths are created on demand when a set of sensor nodes detect an event. It is a purely on-demand design with no proactive routing message exchange.

Reduce the number of packet transmissions to minimum to avoid acoustic collision. To further reduce the number of transmissions, UWD leverages existing in-network processing supports which aggregate homogeneous sensing reports originated from the same set of sources. This mitigates channel contention, especially in stationary scenarios. Nevertheless, the combination of on-demand design and in-network processing is not enough. To reduce the number of on-demand floods and to cope with random node mobility, we use community-to-community forwarding [10], a dynamic unicast-based path management technique, to avoid packet floods (except one or two expensive but indispensable bootstrapping floods). In all cases, UWD seeks to avoid acoustic transmissions unless they are indispensable.

\subsection{Design details}

UWD has 6 packet types: Interest, SinkDiscovery, UnicastReply, Probe, TakeOVERHAPPENS and EVENTREPORT. Only the first two are flooding packets transmitted by MAC broadcast. The others are unicast packets with ACKs similar to 802.11. They are used in the following scenarios.

\subsubsection{Initial floods}

Initial floods are expensive and needed only at the beginning phase of UWD. Initially a sink (command center) floods an INTEREST message to the network. Afterward, there are two possible scenarios depending on whether a sensor node can detect an event within a time threshold $T$ (roughly the estimated time for a node to roam out of a one-hop neighborhood). If the interested event is detected within $T$, a source node can send data to the sink via the shortest latency path. We name this one the Immediate Report Protocol (IRP).

On the other hand, if the interested event happens after time $>T$, routing entries are already stale. The node must again issue a SINKDISCOVERY message (similar to $R R E Q$ message in an on-demand routing protocol) to find the optimal route towards the sink. The sink node will re- 


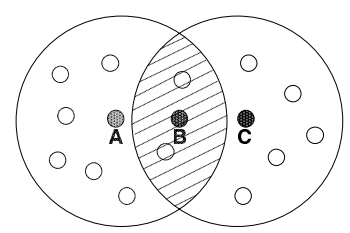

(a) A single forwarding community

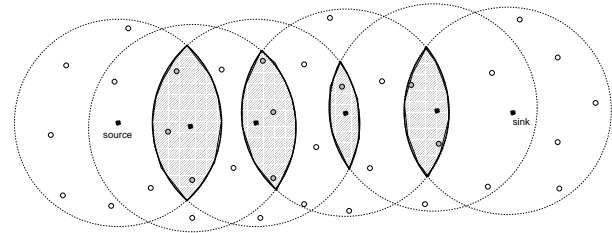

(b) Chain of forwarding communities

Fig. 6. Community-to-community forwarding

spond with a UNICASTREPLY toward the source. As the reporting happens in a delayed fashion, we name this one the Delayed Report Protocol (DRP). Note that overall procedures of IRP and DRP are presented in Figure 4 and Figure 5 respectively.

UWD only has two types of flooding messages: INTEREST and SINKDISCOVERY. In either IRP or DRP, an INTEREST is only sent once (as described below, changes made to the same interest are piggybacked into UNICASTREPLY message from the sink to the source). In DRP, a source proactively sends a SINKDISCOVERY message when it detects an event. Then the sink reactively sends back a UNICASTREPLY. The efficiency of the proposed UWD protocol rests on the fact that UWD limits the use of flooding unless it is necessary (the initial setup). This is achieved by virtue of the community-to-community forwarding approach [10]. For completeness of the paper, in the following subsections we describe key ideas of the community-to-community forwarding.

\subsubsection{Community-to-community forwarding}

This forwarding approach exploits two innate characteristics of wireless sensor networks: (1) redundancy of deployment and (2) omni-directional signal propagation in wireless channels. Figure 6(a) shows the simplest example of a forwarding community between a source $A$ and its sink $C$ that is two-hop away. In a 3-D UWSN, the community area is defined by the intersection of three transmission balls of $A, B$ and $C$. In the figure, node $\mathrm{B}$ is within the intersection of $A$ and $C$ 's radio range while $A$ and $C$ cannot hear each other. In principle, nodes in the intersection (shared region in Figure 6(a)) area form a community and community members (i.e., any nodes in the community area) can relay packets from $A$ to $C$. As depicted in Figure 6(b), this approach can be extended to a chain of forwarding communities along a multi-hop path. Intuitively, in community-to-community forwarding, a community is a "big virtual node" that replaces a single forwarding node in conventional routing schemes.

\subsubsection{Community configuration and re-configuration}

Communities are formed during the first UNICASTREPLY between a source and a sink. In practice, UNICASTREPLY packets are added with a 16-bit hop_count field. The field is reset to 0 at its originator, and is increased by 1 at each stop. Simply by passive and local monitoring, the community members set their community flags upon hearing three consecutive UNICASTREPLY packets of the same interest.

To cope with node mobility, we use proactive probing unicasts to reconfigure the dynamic communities. The source is responsible to send out a PROBE unicast every $T_{p r o b e}$ interval. This is because the source knows whether there are further EVENTREPORTs. The sink responds with 
a UnicastReply. The following example shows that Probe and its UniCastReply can be delivered between the source and its sink if the probing interval $T_{\text {probe }}$ is sufficiently small.

Example 1 Suppose the source uses a controlled PROBE flood (not network-wise flood, also in this example, not unicast) to notify the sink. In such a controlled PROBE flood, only current community members of the interest forward the PROBE packet. This way, the flooding overhead on all other non-community members is saved. Since the needed community flags have been set previously in the initial community configuration or the previous probing rounds, the controlled PROBE flood is approximately equal to a network-wise flood if $T_{\text {probe }}$ is sufficiently small.

Instead of using the controlled flooding described in the example, in the real design PROBE is unicast. From the community protection described below, this protected unicast effectively replaces the controlled flooding design.

If the current forwarder fails to forward a PROBE or UNICASTREPLY packet within $T_{p}$ time, then the current community members seek to take over the current forwarder. Here "take-over" means that a community member competes to forward the PROBE or UNICASTREPLY, with the sender's MAC address set to the community member's and receiver's MAC address unchanged. In other words, it tries to become the current forwarder. Since there are possibly multiple takeover contenders, a collision avoidance process is needed to decrease possible take-over collisions. The take-over trials use a collision avoidance time window at a proper level that is calculated according to the deployment settings (several seconds in Figure 2 and tens of seconds in Figure 3). This way, even though a take-over trial takes relatively long time (compared to radio networks), the trial succeeds with high probability. Once a unicast trial is ACKed by the next stop, all other competing trials stops after the competing senders hear the ACK. Then the unicast take-over trial successfully replaces the original forwarding.

The probing interval $T_{\text {probe }}$ is adapted with respect to network dynamics. Whenever a takeover action succeeds, the taking-over node sends a short TAKEOVERHAPPENS report to the source. $T_{\text {probe }}$ is initialized to be $R / v$ where $R$ is the well-known one-hop transmission range and $v$ is the estimated average node mobility speed. The quantity $R / v$ estimates the time of next link outage due to node mobility. The source decreases its $T_{\text {probe }}$ by a larger value (e.g., $100 \mathrm{~ms}$ ) upon receiving every such take-over report, and increases $T_{\text {probe }}$ by a smaller value (e.g., $10 \mathrm{~ms}$ ) if no take-over report is received within the most recent second. As frequent take-over actions indicate more network dynamics, the simple heuristic scheme seeks to maintain fresher communities by launching more probing requests. Meanwhile it also seeks to decrease probing overhead when the communities en route are relatively stable. As a result, even if the number of SINKDISCOVERY floods per interest is not ideal in the real world ( 1 in the ideal IRP or 2 in the ideal DRP), this heuristic community-based scheme significantly reduces the flooding frequency.

\subsubsection{In-network processing}

When a SinkDiscovery, Probe or EventReport is forwarded towards the sink, it is often the case that other sensor nodes nearby the Center of Stimulus (CoS) of the event also detect the same interested event and try to send the same message to the sink. In UWD, multiple SINKDISCOVERY or EVENTREPORT of the same interest are aggregated together if their timestamps are within a time threshold $t$ (which is proportional to the motion speed of the interested target). The aggregator node remembers the merged incoming links in its soft state. Then the later UNICASTREPLY from the sink on the reverse direction will be replicated to the previously merged links by the aggregator. In addition, any PROBE message is aggregated into ongoing 


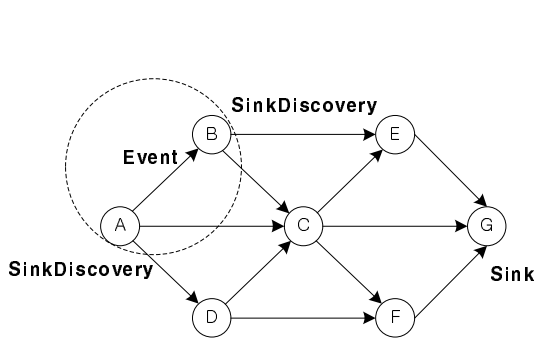

(a) Multiple sources detect the same event

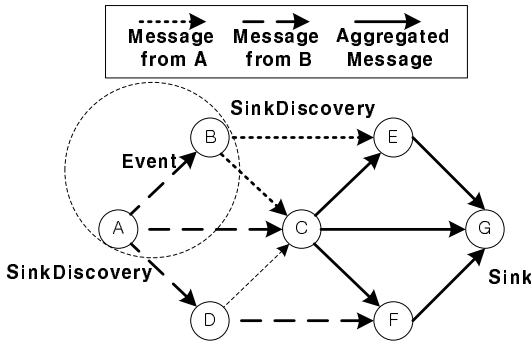

(b) SINKDISCOVERY aggregation

Fig. 7. In-network processing

EVENTREPORT, and any TAKEOVERHAPPENS report is aggregated into ongoing UNICASTREPLY whenever possible.

\section{Analysis of Under-Water Diffusion}

In this section we prove that UWD only has negligible amount of on-demand floods.

\subsection{Underlying network model}

We divide the network area into a large amount of small (virtual) tiles, so that the tile size is even smaller than the physical size of the smallest network member. This way, each tile is either empty, or is occupied by a single node. Also because the network area is much larger than the sum of all mobile nodes' physical size, the probability that a tile is occupied by a mobile node is very small.

Now a binomial distribution $B(\eta, p)$ defines the probabilistic distribution of how these tiles are occupied by each mobile ad hoc node. Here $\eta$, the total number of "positions", is very large; and $p$, the probability that a tile is occupied by the single node, is very small. When $\eta$ is large and $p$ is small, it is well-known that a binomial distribution $B(\eta, p)$ approaches Poisson distribution with parameter $\rho_{1}=\eta \cdot p$. Hence this binomial spatial distribution is translated into a spatial Poisson point process [3] to model the random presence of the network nodes. In other words, $\rho_{1}$ can be treated as a mobile node's arrival rate of each standing "position". Moreover, suppose that $N$ events occur in area $\mathcal{A}$ (here an event is a mobile node's physical presence), $\rho_{N}=\frac{N}{\mathcal{A}}$ (where $\rho_{N}=N \cdot \rho_{1}$ if $N$ nodes roam independently and identically distributed) is equivalent to a random sampling of $\mathcal{A}$ with rate $\rho_{N}$.

Let $x$ denote the random variable of number of mobile nodes in any network area concerned:

- (Uniform $\left.\rho_{1}\right)$ the probability that there are exactly $k$ nodes in a specific area $\mathcal{A}^{\prime}$ following a uniform distribution model is

$$
\operatorname{Pr}[x=k]=\frac{\left(N \cdot \rho_{1} \cdot \mathcal{A}^{\prime}\right)^{k}}{k !} \cdot e^{-N \cdot \rho_{1} \cdot \mathcal{A}^{\prime}} .
$$

- (Non-uniform $\rho_{1}$ ) More generally, in arbitrary distribution models including non-uniform models, the arrival rate is location dependent. The probability that there are exactly $k$ nodes in a specific area $\mathcal{A}^{\prime}$ is 


$$
\operatorname{Pr}[x=k]=\iint_{\mathcal{A}^{\prime}}\left(\frac{\left(N \cdot \rho_{1}\right)^{k}}{k !} \cdot e^{-N \cdot \rho_{1}}\right) \mathrm{d} \mathcal{A} .
$$

\subsection{Negligible on-demand SINKDISCOVERY floods}

The number of packet transmissions in UWD is computed as the sum of the following transmissions: (1) the initial INTEREST flood; (2) the initial SINKDISCOVERY flood if in DRP mode; (3) the source-initiated Probe unicasts plus the take-over unicast trials, if there is any; (4) the coming-back UNICASTREPLY unicasts plus the take-over unicast trials, if there is any; (5) the EVENTREPORTs. The first factor (1) is application driven and cannot be suppressed at the sink(s). The last factor (5) is application driven and cannot be suppressed at the sources. But the innetwork processing will further reduce the number of (2) SinkDiscovery, (3) PROBE and (4) EVENTREPORT packets that are of the same interest and transmitted in adjacent time. The (3) and (4) unicast overheads are paid to trade with the (2) flooding overheads. Here we analyze the efficacy of this trade-off. Suppose the geometric size of an average forwarding community is $\mathcal{A}_{\text {avg }}$, the probability that there are exactly $k$ nodes in the average forwarding community is

$$
\operatorname{Pr}[x=k]=\iint_{\mathcal{A}_{\text {avg }}} \frac{\left(N \cdot \rho_{1}\right)^{k}}{k !} \cdot e^{-N \cdot \rho_{1}} \mathrm{~d} \mathcal{A}
$$

In UWD, the failure probability of PROBE or UNICASTREPLY packet forwarding at each hop is when the forwarding community is empty:

$$
P_{\text {failhop }}=\operatorname{Pr}[x=0]=\iint_{\mathcal{A}_{\text {avg }}} e^{-N \cdot \rho_{1}} \mathrm{~d} \mathcal{A} .
$$

The mobility PDF $\rho$ is arbitrary in our study, thus could be location dependent and becomes a function of the location area $\mathcal{A}$. Therefore, double integrals must be used here (or triple integrals in case of 3D scenarios). Fortunately, because $e^{x}$ is a fixed point in differential and integral calculus, such differentials and integrals do not change the magnitude of order, that is, $\frac{\mathrm{d} e^{x}}{\mathrm{~d} x}=e^{x}$ and $\int e^{x} \mathrm{~d} x=e^{x}+C=O\left(e^{x}\right)$. This concludes that the probability of route discovery or probing failure per hop/step $P_{\text {failhop }}$ is negligible with respect to the network scale $N$.

The failure probability of mobile route maintenance, that is, the failure probability of a probing source fails to receive the coming-back UNICASTREPLY, is

$$
P_{\text {failprobe }} \leq 1-\left(1-P_{\text {failhop }}\right)^{2 \cdot N}
$$

where $2 N$ is for the worst case, when all mobile nodes organized into a linear chain topology, thus the route discovery procedure will end in $2 \cdot N$ hops. Then let's prove that $P_{\text {failprobe }}$ is also negligible.

By previous conclusions, $P_{\text {failhop }}$ is negligible, which must be asymptotically less than any given $\frac{1}{(2 N+1) \cdot q(N)}$, where $q(N)$ is a positive polynomial and $(2 N+1) \cdot q(N)$ is also a positive polynomial. In other words, there exists a positive integer $N_{c}>0$, such that $P_{\text {failhop }}<\frac{1}{(2 N+1) \cdot q(N)}$ for all $x>N_{c}$. Then we have

$$
\left(1-P_{\text {failhop }}\right)^{2 N}>\left(1-\frac{1}{(2 N+1) \cdot q(N)}\right)^{2 N}>e^{-\frac{1}{q(N)}}
$$


since $\left(1-\frac{1}{x}\right)^{x-1}>e^{-1}$ for all $x>1$.

According to Lagrange mean value theorem, for a function $f(x)$ continuous on $[a, b]$, there exists a $c \in(a, b)$ such that $f(b)=f(a)+f^{\prime}(c) \cdot(b-a)$ for $0<a<b$. Then let $f(x)=e^{-x}$, there exists a $\xi \in(0, z)$, such that $e^{-z}=1+\left(-e^{-\xi}\right) \cdot z>1-z$. Thus we have

$$
\left(1-P_{\text {failhop }}\right)^{2 N}>e^{-\frac{1}{q(N)}}>1-\frac{1}{q(N)} .
$$

Therefore, for any polynomial $q(N)$ and sufficiently large $N$,

$$
P_{\text {failprobe }}=1-\left(1-P_{\text {failhop }}\right)^{2 N}<\frac{1}{q(N)} .
$$

When unicast probing works, there is no need to issue highly expensive SINKDISCOVERY floods to find a path to the mobile sink. In summary, only with the negligible ${ }^{2}$ probability $P_{\text {failprobe }}$, an expensive network SINKDISCOVERY flood is invoked in UWD to ensure mobile ad hoc routing.

\section{Simulation Study}

In this section, we perform a simulation study to validate our protocol. We describe details of underwater simulation environments. After explaining metrics of interest, we evaluate how Under-Water Diffusion performs compared to Directed Diffusion.

\subsection{Simulation Environment}

The underwater acoustic channel is significantly different from wireless radio channel and thus in this subsection we describe how we enhanced $n s-2$ simulator ${ }^{3}$ to support underwater simulations. To this end, we modified both physical and MAC layers of the wireless network simulation stack of $n s-2$.

In the physical layer we modified the signal propagation model in terms of propagation speed and transmission loss. First, the speed of sound in underwater is a function of temperature and pressure as presented in [9]. For routine estimations of a shallow UWSN, however, a speed of sound of $1500 \mathrm{~m} / \mathrm{s}$ is adequate. Therefore, we simply changed SPEED_OF_LIGHT to $1500 \mathrm{~m} / \mathrm{s}$. Second, the intensity of the acoustic wave signal is mainly reduced with increasing range due to spreading effect [9]; thus, we simulate spreading effects.

For the MAC layer we used the simple MAC defined in $n s-2$. Since large propagation latency of acoustic waves makes carrier sensing ineffective, we removed the carrier sensing part of the simple MAC, and non-slotted ALOHA. The maximum collision avoidance time is predefined based on network density and in our simulations, we used 1 second. The data rate is set to $7 \mathrm{kbps}$ which is the payload data rate of an off-the-shelf acoustic modem (LinkQuest UWM1000 [11]) and transmission radius was set to $100 \mathrm{~m}$. In our simulations, we simply assume that sensors are randomly moving and thus use a random walk mobility model with average speed of $1.5 \mathrm{~m} / \mathrm{s}$.

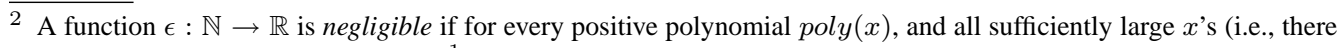
exists $N_{c}$, for all $\left.x>N_{c}\right), \epsilon(x)<\frac{1}{\text { poly }(x)}$.

3 http://www.isi.edu/nsnam/ns/
} 


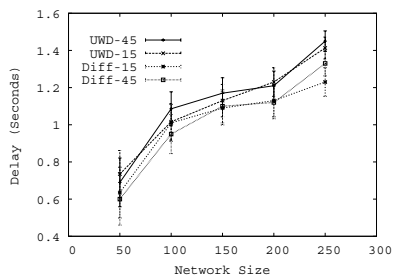

(a) Average delivery delay

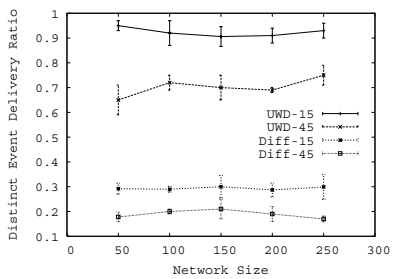

(b) Average delivery ratio

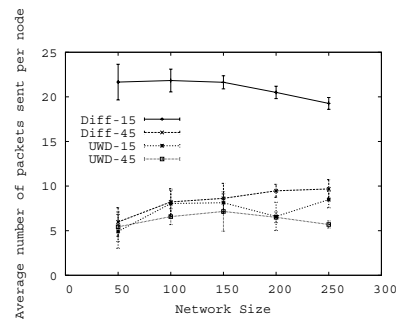

(c) Per node overhead

Fig. 8. Performance comparison between Directed Diffusion and Under-Water Diffusion

\subsection{Methodology}

We compare our protocol with Directed Diffusion using the following metrics: average event delivery delay, distinct-event delivery ratio, and average overhead. Average delay measures the average event latency that is the time between sending an event at a source and receiving the event at a sink. This metric is used to measure how timely the report is to (assuming that the report is time sensitive). Distinct-event delivery ratio is the ratio of the total number of events received by the sink to the number of events sent by the source(s). This metric shows how the proposed protocol reacts to the node mobility. Average overhead measures the average number of packets sent per node. Since a major source of overhead is flooding, this metric is used to show how our protocol limits the use of flooding compared to Directed Diffusion.

To evaluate such metrics, we set protocols as follows. In the case of Directed Diffusion, interests and exploratory messages were periodically sent to handle mobility. We used two periods for both interests and exploratory events. Note that exploratory events are used for setting up a new path (through positive reinforcement) and interests are used for creating forward gradients to the sink. This allows us to see the impact of the interest period (IP) and the exploratory event period (EP). In the simulation, the IP was either $15 \mathrm{~s}$ or $45 \mathrm{~s}$ and the EP was either $15 \mathrm{~s}$ or $45 \mathrm{~s} .{ }^{4}$ Because an exploratory event uses paths created by interests, we used only combinations of (IP$15 s, E P-15 s)$ and $(I P-45 s, E P-45 s)$ which we call Diff- 15 and Diff-45 respectively. We used the window for the negative reinforcement to be 5 seconds. In the case of UnderWater Diffusion, an INTEREST message was broadcast once at the very beginning. For the period of a PROBE message, we used the same period as Directed Diffusion for the fair comparison and thus we used 15 s and 45 s intervals which we call $U W D-15$ and $U W D-45$ respectively.

To study the performance as a function of network size, we generated various sizes of sensor fields. To this end, for each experiment we use four different sizes of sensor fields ranging from 50 to 250 nodes in increments of 50 nodes. As a default, we deployed 50 nodes in a field of a $500 \mathrm{~m} \times 500 \mathrm{~m}$ square area. For other sizes of fields, we kept the same network density and scaled the size of a sensor field. For instance, in the case of the network size of 200 nodes, we scaled the field to $1000 \mathrm{~m} \times 1000 \mathrm{~m}$ square area. Event sources were located in a $100 \mathrm{~m} \times 100 \mathrm{~m}$ square area of the top left corner of the network and a sink is randomly selected from the network. Sources generated an event every 5 seconds and the size of a message was 128B. We ran simulations for 200 seconds and each metric was measured by averaging 30 runs.

\footnotetext{
${ }^{4}$ We can further shorten the period, but we should note that such frequent use of flooding in underwater could make the whole network collapse.
} 


\subsection{Evaluation}

The average delay for an event is shown in Fig. 8(a). The graph shows that the average delay increases in both protocols as we enlarge the network size, which is done by keeping the same network density and scaling the size of a field. Thus, due to large propagation latency and low data rate, the longer the average distance between a source and a sink, the longer the average delay. It is interesting to note that since a packet could be forwarded with help of communities, Directed Diffusion exhibits shorter average delay than UWD. For each takeover, a community member must wait $2 \cdot T_{p}=0.134 \mathrm{~s}$ and thus the more the takeovers, the longer the average delay. In reality, for a given packet, the number of experienced takeovers is usually small and thus this will not harm the overall performance of our protocol. If an application is delay sensitive, we can use the following heuristics. While a packet is forwarded toward the sink, each forwarding node can check a maximum allowable jitter. If the current delay value is higher than the maximum allowable jitter, a forwarding node simply discards the message. In our study, after removing outliers, we are able to achieve roughly the same average delay as Directed Diffusion, but this, in fact, decreases the average delivery ratio; however, the impact is less than $10 \%$ of the original delivery ratio.

Fig. 8(b) shows the average delivery ratio as a function of network size. Unlike a Ground Sensor Network (GSN) where an event delivery ratio is close to one, mobility in a UWSN incurs packet losses, thus making the ratio less than one. While the delivery ratio of UWD with 15 s probing interval is above $90 \%$, that of Directed Diffusion is less than 30\%. In most cases, community-based forwarding takes care of node mobility, but there could be the cases that before a route management packet reconfigures a path, the path could be broken. If that happens, we must broadcast SINKDISCOVERY again. In our simulation, route management is carried out every $15 \mathrm{~s}(U W D-15)$ or $45 \mathrm{~s}(U W D-45)$ and UNICASTREPLY timeout value is $5 \mathrm{~s}(<$ total $20 \mathrm{~s}$ or $50 \mathrm{~s})$. In addition, we need to flood SINKDISCOVERY and must receive UNICASTREPLY again $(<$ total 10s). This roughly takes a total of 30s or $60 \mathrm{~s}$, and thus we are losing up to $6(U W D-15)$ or 12 (UWD-45) packets. With 15s period of route management, we can achieve above $90 \%$, but if we set the period as $10 \mathrm{~s}$, then we achieve nearly $100 \%$ (it is not shown in the simulation results). As shown in the figure, in contrast, if we set the period as $45 \mathrm{~s}$ period, then the event delivery ratio is decreased to around $60 \%$.

Finally, Fig. 8(c) shows per node overhead as a function of network size. Unlike Directed Diffusion where its heavy use of flooding to handle mobility incurs considerable per node overhead, UWD reduces such overhead with help of a community based forwarding mechanism. For instance, Diff-15 incurs almost 4 times larger overhead than UWD-15. In the case of Directed Diffusion, we can roughly estimate the number of floods due to its periodical flooding. During 200 seconds of simulation time, roughly Diff- 15 and Diff- 45 use flooding 26 (Interest $\times 13$, Exploratory $\times 13$ ) and 8 (Interest $\times 4$, Exploratory $\times 4$ ) times respectively. In contrast, UnderWater Diffusion utilizes unicast probing (UNICASTPROBE), thus minimizing the number of flooding. In our simulations, the average number of floods was less than 4 even in the case of $U W D-45$. Note that in UWD extra packets are sent only when a packet is forwarded with help of community members, and extra floods happen only when a source node fails to receive the UNICASTPROBE packet. Therefore, we conclude that the shorter the route management period, the better the relative effectiveness of our protocol.

In summary, our simulation results confirm that reducing the number of floods is a key design choice in designing underwater sensor network protocols. We show that in underwater, Directed 
Diffusion which manages mobility using periodical flooding, is less efficient because of its heavy use of flooding. Our proposed protocol, on the other hand, by limiting flooding, we can increase overall delivery ratio and reduce per node overhead.

\section{Related work}

Small-scale Underwater Acoustic Networks (UAN) have been explored in [18,21]. In [18], each node maintains a neighbor table and feeds the table to a centralized sink, who then build a routing tree. In a sensor network with random node mobility, this design has to use a proactive neighbor detection protocol to constantly take fast snapshots of the mobile network topology. In [21], the sink periodically sends out a topology discovery message to acquire the current network topology. Periodical floods of route discovery messages will incur severe traffic and thus disrupt the efficiency of the protocol. In contrast, UWD prohibits proactive routing message exchange and minimizes the use of on-demand floods in order to decrease the probability of collisions.

Position-based routing (or geo-routing) for static UWSN is investigated in $[22,16]$. In VectorBased Forwarding (VBF) [22], nodes close to the straight line between source and destination are eligible to forward packets, and thus, multiple nodes can forward a packet. Since many nodes can potentially forward packets in a dense network, a VBF forwarder uses a localized metric based on distance to find the best forwarder. Pompili et al. proposed localized, energy-efficient forwarding algorithms such that a forwarding node selects the best next hop, the transmission power, and the FEC code rate for each packet, with the objective of minimizing the energy consumption [16]. These schemes assume that there exists localization. However, providing accurate localization for processing depraved UW nodes is nontrivial. Since GPS signals cannot reach underwater, the most promising approach is to use Time-of-Arrival (TOA) of acoustic signals. But this requires sophisticated signal processing since the underwater environment with motion of water and variation in temperature and pressure affects the speed of acoustic signal. If nodes are mobile, localization will incur more message exchanges, thus further limiting the feasibility of accurate localization. Moreover, voids could be found when sensors are not deployed uniformly. In this case a forwarding node must rely on face-routing, but planarization with asymmetric links and location errors incurs considerable overheads [8].

Salva-Garu et al. [17] proposed a decentralized multiple access scheme based on clustering for an autonomous network of UUVs (Underwater Unmanned Vehicles). TDMA is used for intra-cluster communication and CDMA for inter-cluster communication. Nodes in the network know their positions (from cables). Based on the critical geo-information, clusters are formed and maintained, then TDMA slots are allocated and CDMA codes are distributed. However, TDMA is more suitable to stationary, tethered or GPS-equipped networks rather than to mobile, tetherless and GPS-free networks because a pre-requisite of TDMA in a mobile tetherless neighborhood is to employ a proactive neighbor detection protocol to maintain up-to-date one-hop neighborhood knowledge. Moreover, the code orthogonality is quickly exhausted in the heavilycontended narrow-band acoustic channel and thus, shared medium random access protocols such as ALOHA can be used as the last resort. Therefore, UWD relies on unicast transmissions using non-slotted ALOHA, which is known to be collision resistant with community based forwarding [10] and thus, this complements CDMA's channel orthogonality supports.

UWD is best suited for real-time surveillance applications such as submarine detection. In the case of delay-tolerant applications (e.g., estuary monitoring), we could consider various routing schemes in delay-tolerant networks (DTN). For instance, Vasilescu et al. proposed an underwater 
data harvesting system where a data mule is roaming to harvest data from static sensors [19]. They used acoustic communications for localization and optical communications for data pulling from static nodes. Similarly, Magistretti et al. proposed Delay-tolerant Data Dolphin (DDD) that exploits the mobility of a small number of capable collector nodes (namely dolphins) to pull data from its one-hop neighbors [12].

\section{Summary}

We have proposed a new Under-Water Sensor Network (UWSN) architecture. Unlike the existing Underwater Acoustic Networks (UAN), this new architecture uses large number of unmanned low-cost sensor nodes to locally monitor and report non-accessible underwater events in a time-critical manner. However, due to the large propagation latency and low bandwidth of the challenging Under-Water Acoustic (UW-A) channel, new models and protocols are needed at most protocol stack layers. In this paper we study multi-hop packet delivery, an essential network component including mobile ad hoc routing and data centric in-networking services. We show that flooding cannot be both reliable and efficient. Because current GPS-free routing and diffusion schemes rely on (network-wise or controlled) floods, a direct application of these schemes fails with high probability. To answer this challenge, Under-Water Diffusion (UWD) is proposed. UWD takes a minimalist's view. It assumes homogeneous GPS-free nodes (i.e., without the help of heterogeneous backbone nodes with abundant resource), random node mobility and no proactive design. This is completely different from terrestrial sensor networks, where each of these non-minimalist features can be used to gain performance. In UWD, we seek to answer the multihop routing challenge without breaking the constraints. We use community-based forwarding and unicast PROBE flows to cope with node mobility and to reduce the number of floods per interest to one or two expensive initial floods. We rely on MAC-unicast transmissions on the optimal paths rather than MAC-broadcast transmissions in flooded areas. We avoid the use of proactive exchange. This way, UWD is able to minimize the number of floods and the number of various other packet transmissions to avoid looming acoustic collisions. Our experimental results justify the effectiveness and efficiency of our design.

\section{References}

[1] J. Broch, D. A. Maltz, D. B. Johnson, Y.-C. Hu, and J. Jetcheva. A Performance Comparison of Multi-Hop Wireless Ad Hoc Network Routing Protocols. In ACM MOBICOM, pages 85-97, 1998.

[2] N. Bulusu, J. Heidemann, and D. Estrin. GPS-less Low Cost Outdoor Localization for Very Small Devices. IEEE Personal Communications Magazine, 7(5):28-34, 2000.

[3] N. Cressie. Statistics for Spatial Data. John Wiley and Sons, 1993.

[4] C. Intanagonwiwat, R. Govindan, and D. Estrin. Directed Diffusion: A Scalable and Robust Communication Paradigm for Sensor Networks. In ACM MOBICOM, pages 56-67, 2000.

[5] D. B. Johnson and D. A. Maltz. Dynamic Source Routing in Ad Hoc Wireless Networks. In T. Imielinski and H. Korth, editors, Mobile Computing, volume 353, pages 153-181. Kluwer Academic Publishers, 1996.

[6] A. Kaya and S. Yauchi. An Acoustic Communication System for Subsea Robot. In Oceans'89, pages 765-770, 1989.

[7] D. B. Kilfoyle and A. B. Baggeroer. The State of the Art in Underwater Acoustic Telemetry. IEEE Journal of Oceanic Engineering, OE-25(1):4-27, January 2000.

[8] Y.-J. Kim, R. Govindan, B. Karp, and S. Shenker. Lazy Cross-Link Removal for Geographic Routing. In SenSys'06, 2006.

[9] L. E. Kinsler, A. R. Frey, A. B. Coppens, and J. V. Sanders. Fundamentals of Acoustics. John Wiley and Sons, 1999. 
[10] J. Kong, X. Hong, Y. Yi, J.-S. Park, J. Liu, and M. Gerla. A Secure Ad-hoc Routing Approach using Localized Self-healing Communities. In ACM MOBIHOC'05, pages 254-265, 2005.

[11] LinkQuest. Underwater Acoustic Modems UWM2000 Specifications. http://www. link-quest.com/ html/uwm2000.htm.

[12] E. Magistretti, J. Kong, U. Lee, M. Gerla, P. Bellavista, and A. Corradi. A Mobile Delay-tolerant Approach to Long-term Energy-efficient Underwater Sensor Networking. In WCNC'07, 2007.

[13] D. Maltz, J. Broch, J. Jetcheva, and D. Johnson. The Effects of On-Demand Behavior in Routing Protocols for Multi-Hop Wireless Ad Hoc Networks. IEEE Journal on Selected Areas in Communication, 17(8):1439-1453, 1999.

[14] R. Motwani and P. Raghavan. Randomized algorithms. Cambridge University Press, 1995.

[15] C. E. Perkins and E. M. Royer. Ad-Hoc On-Demand Distance Vector Routing. In IEEE WMCSA'99, pages 90-100, 1999.

[16] D. Pompili, T. Melodia, and I. F. Akyildiz. Routing algorithms for delay-insensitive and delay-sensitive applications in underwater sensor networks. In MobiCom'06, 2006.

[17] F. Salva-Garau and M. Stojanovic. Multi-Cluster Protocol for Ad Hoc Mobile Underwater Acoustic Networks. In IEEE OCEANS'03 Conference, 2003.

[18] E. M. Sozer, M. Stojanovic, and J. G. Proakis. Undersea Acoustic Networks. IEEE Journal of Oceanic Engineering, OE-25(1):72-83, January 2000.

[19] I. Vasilescu, K. Kotay, D. Rus, P. Corke, and M. Dunbabin. Data Collection, Storage and Retrieval with an Underwater Optical and Acoustical Sensor Network. In SenSys'05, 2005.

[20] L. F. M. Vieira, J. Kong, U. Lee, and M. Gerla. Analysis of Aloha Protocols for Underwater Acoustic Sensor Networks. In The First ACM International Workshop on UnderWater Networks (WUWNet), 2006.

[21] G. G. Xie and J. Gibson. A Networking Protocol for Underwater Acoustic Networks. Technical Report TR-CS-0002, Department of Computer Science, Naval Postgraduate School, December 2000.

[22] P. Xie, J.-H. Cui, and L. Lao. VBF: Vector-Based Forwarding Protocol for Underwater Sensor Networks . In Networking'06, 2006.

\section{Acknowledgement}

This work was supported in part by the National Science Foundation under Grant No. 0520332 and the US Army under MURI award W911NF-05-1-0246. The research is continuing through participation in the International Technology Alliance sponsored by the U.S. Army Research Laboratory and the U.K. Ministry of Defence under Agreement Number W911NF-06-3-0001. 